\title{
Scalable Schedule Routing and Minimizing Delay in Tolerant Networks
}

\author{
Dhanasekaran. $\mathrm{P}^{1}$, Ramani. $\mathrm{R}^{2}$ \\ 1 P.G.Scholar, Department of Computer Science \& Engineering, PSR Engineering College, Sivakasi, India. \\ 2 Assitant Professor, Department of Computer Science\& Engineering, PSR Engineering College, Sivakasi, \\ India.
}

\begin{abstract}
There are many routing strategies for message delivery in Delay Tolerant Networks. Among them Multicopying routing strategies have been considered the most applicable DTNs. Epidemic routing and two-hop forwarding routing are the two best approaches of DTN. These two approaches belong to multicopying routing strategies as they allow multiple message replicas to increase message delivery delay. This is an advantage, but it might cause expense of additional buffer space and bandwidth overhead. Hence the message scheduling strategy should determine which messages should be dropped in case if buffer is full. Thus, this work deals with a new message scheduling framework for epidemic and two hop forwarding routing in DTNs, such that the decision for forwarding/dropping can be made at a node during each contact for either optimal message delivery ratio or message delivery delay. Extensive simulation results show that this message scheduling framework can achieve better performance.
\end{abstract}

Keywords: Routing, buffer management, message scheduling, DTN.

\section{Introduction}

Delay Tolerant Networks (DTN) is one of the emerging types of networks whose distinguishing characteristic is that communications between two nodes in a DTN take prolonged period of time because of lack of continuous connection. DTNs were initially designed to operate over extreme distances encountered in space communications where the long latency in communications inevitably causes TCP connections in the network to break down. As the TCP communication breaks down in a DTN, a Bundle Protocol can be used to handle the underlying communication with the remote party using node-to-node custody transfers. This protocol also allows the DTN to span multiple heterogeneous networks and allows the DTN protocols to be separated from the naming and addressing schemes used in the underlying networks. However, the discontinuous connection problem due to the cosmic distances can also occur over shorter terrestrial distances due to lack of infrastructure. For example, many people living in remote rural areas and in developing countries do not have access to the Internet.

To realize data communication in such cases, it requires hardware to store large amounts of data, and also requires special media to transfer data between the nodes. Therefore, in a terrestrial context, a DTN can be appropriately regarded as a group of highly disconnected networks/regions where the communication environment is characterized by high error rates, long delays and sporadic connectivity with the outside world. An approach to transfer the data between the disconnected network regions is by making use of message ferry which travels on arbitrary paths between the regions and has a data storage buffer which can be used to keep messages. Each region has a fixed gateway node which handles the communication needs of incoming and outgoing traffic for the hosts within the region. When a ferry arrives at a region, it contacts the gateway node, and during the contact time it will unload to the gateway the messages that are destined to this region, and load from the gateway the messages that this region wants to send to other regions.

\section{Problem Statement}

The buffering and forwarding unlimited number of messages may also cause intolerable resources and nodal energy consumption; and it is imperative to set up buffer limitations at the DTN nodes to better account for the fact that each node could be a hand-held and battery-powered device with stringent limitations on buffer space and power consumption. With such buffer limitations at the DTN nodes, message drop/ loss could happen due to buffer overflow. This leads to a big challenge in the implementation of most previously reported DTN routing scheme.

\section{Proposed System}

New utility-based message scheduling mechanism that incorporates with DTN message forwarding, where per-message utility is determined to optimize either message delivery ratio or delivery delay. Developing a novel estimation approach for network global knowledge to facilitate decisions on which message should be 
forwarded/dropped when the buffer of the encountered node is full. Evaluating and comparing the proposed scheme with counterparts, and gaining understanding on its tradeoff between computation complexity and performance improvement.

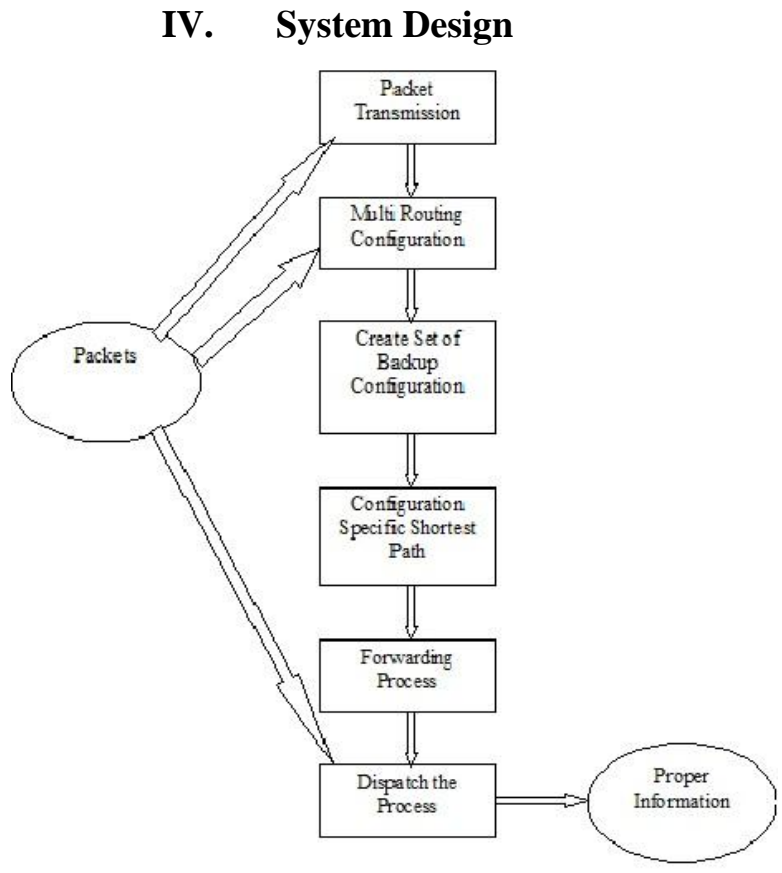

Fig.4.1 Packet Forwarding Process

\section{System Implementation}

The proposed system consists of four main modules. They are

- Client and Router Designing

- Selection of two nearby nodes

- Selection of Congestion-free nearby nodes

- Transmitted data tracing and report generation

\subsection{Client and Router Designing}

The client and router design is used to arrange the users and the routers. MIM is used to avoid the traffic in networks and the packets are sent and receive using the signal strength, in this there are multiple node is available and if the first node strength is low means it will be automatically switched to the other node, during the packets switching at the time it is possible to hack the packets so I have to use adaptive opportunistic routing and to avoid the intruder and packet loss. And in this module arranging the client and the routers for transmit the packets between the one source and destination.

MIM is empowering because it enables a receiver to decode a SOI, even if the SOI arrives after the receiver has already locked on to the interference. Of course, the required signal-to-interference-plus-noise ratio (SINR) is higher for relocking onto the new signal. Conversely, if the SOI arrives earlier than the interference, the reception with MIM-capable hardware is same as traditional reception.

\subsection{Selection of two nearby nodes}

The selection of two nearby nodes is used to send and receive the packets between the sender and receiver using the signal strength. A MIM technique analyzes the signal strength and transmits the packets between the source and destination. And the nodes will be automatically assigning depends the signal strength using the MIM technique. The packets will be sent to the newly assigning nodes depending upon the signal strength and the packets will be able to loss when the node will be switching and using the adaptive opportunistic routing for avoiding the packet loss and to increase the security. 


\subsection{Selection of Congestion-free nearby nodes}

Congestion in a network may occur at interval time when the incoming traffic is larger than the capacity of the network. This network congestion can several increase delay and packet loss and cut the network throughput. Congestion control refers to techniques that can keep away from congestion before it happens or recovery after it happens. The main aim of congestion control is to lower the End to End delay and reduced packet lost caused by network congestion and offer better performance of the network.

In wire line networks, congestion control is employed at the transport layer and it is independent from the functionality of other layers However, these congestion control techniques do not apply directly to ad hoc networks, because the ad hoc network is challenged by a limited wireless bandwidth, power constraints and route failures, due to node mobility and limited buffer size. The final result is a high packet-loss rate, re-routing instability, loss of energy, bandwidth and retransmission of lost packets, which implies that more packets are transmitted in the network. These delays and packet losses are not originated by network congestion, but this can be misinterpreted as congestion losses.

\subsection{Transmitted data tracing and report generation}

Generated the report that is, what are all the packets transmit between the sender and receiver. And will tracing the packets i.e., the packets is using which node, signal strength of the node and the strength of the packets. When the packets are sent between the sender and the receiver the details will be automatically traced and the results will be generated. Sometimes the intruder will be enter and the intruder will be able to loss the packets or destroy the nodes, so this thing also will be traced. It is also used for security purpose and to avoid hacking.

VI. Experimental Results

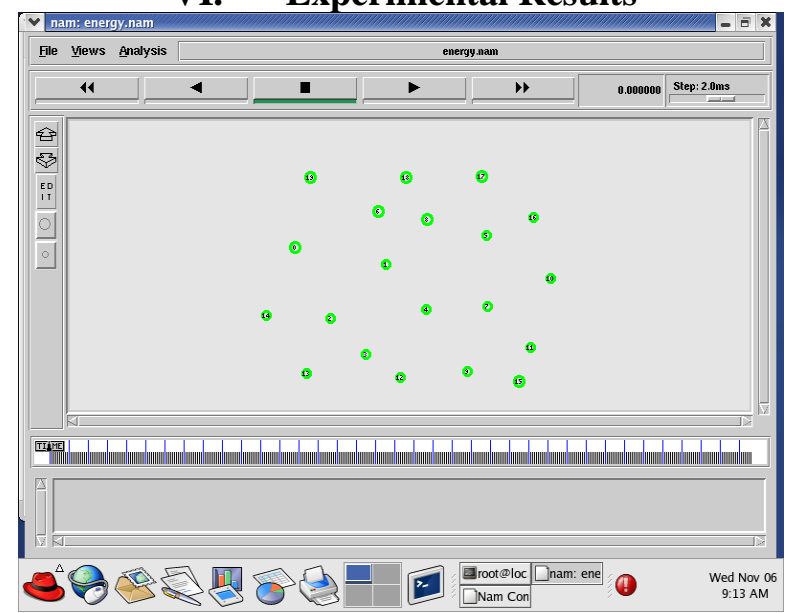

Fig 6.1 Initialization of nodes



Fig 6.2 Packets forwarding and dropping 


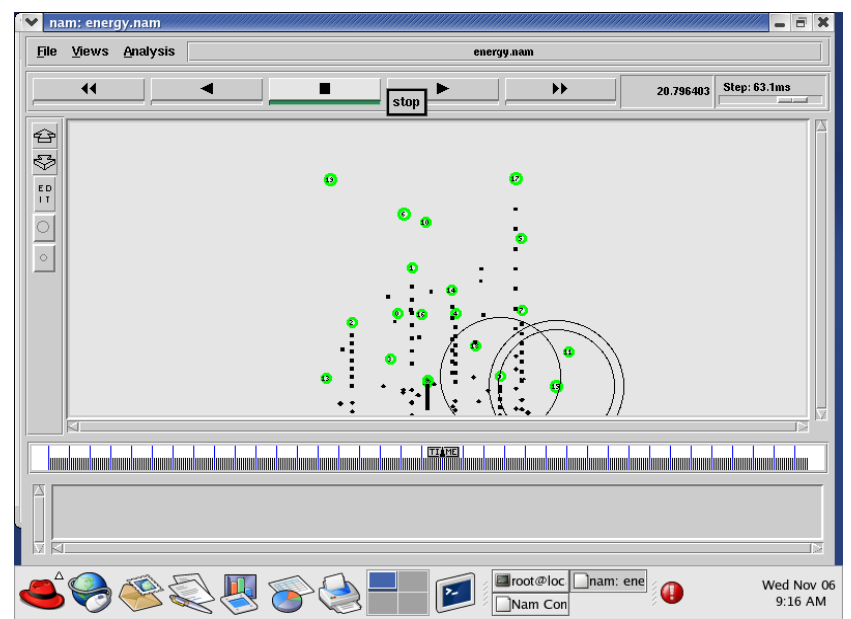

Fig 6.3 Mobility of nodes



Fig 6.4 Simulation time vs delay

\section{Conclusion}

The protocol in designing a communication stacks such that state information flows throughout the stack has been investigated. Simulations based on NS-2 have been carried out to verify the performance of the proposed scheme. Experimental results confirm that the proposed scheme outperforms all other schemes, including IEEE 802.11 distribution by the selective route mechanism in terms of the packet delivery ratio, the average end-to-end delay, and the normalized routing overhead energy.

\section{References}

[1] Ahmed Elwhishi, Pin-Han Ho, K. Naik, and Basem Shihada "A Novel Message Scheduling Framework for Delay Tolerant Networks Routing". IEEE TRANSACTIONS ON PARALLEL AND DISTRIBUTED SYSTEMS, VOL. 24, NO. 5, MAY 2013

[2] Member, IEEE, Thierry Turletti, Member, IEEE, and Katia Obraczka, Member, IEEE , " Routing in Delay-Tolerant Networks Comprising Heterogeneous Node Populations Thrasyvoulos Spyropoulos", IEEE TRANSACTIONS ON MOBILE COMPUTING, VOL. 8, NO. 8, AUGUST 2009

[3] Cong Liu and Jie Wu,Fellow "Scalable Routing in Cyclic Mobile Networks". IEEE TRANSACTIONS ON PARALLEL AND DISTRIBUTED SYSTEMS, VOL. 20, NO. 9, SEPTEMBER 2009

[4] Eyuphan Bulut, Member, IEEE, Zijian Wang, and Boleslaw Karol Szymanski, Fellow "Cost-Effective Multiperiod Spraying for Routing inDelay-Tolerant Networks", IEEE. IEEE/ACM TRANSACTIONS ON NETWORKING, VOL. 18, NO. 5, OCTOBER 2010

[5] Yong Li, Student Member, IEEE, Yurong Jiang, Depeng Jin, Member, IEEE, Li Su, Lieguang Zeng, and Dapeng (Oliver) Wu, Senior Member, IEEE "Energy-Efficient Optimal Opportunistic Forwarding for Delay-Tolerant Networks". IEEE TRANSACTIONS ON VEHICULAR TECHNOLOGY, VOL. 59, NO. 9, NOVEMBER 2010

[6] Giovanni Resta and Paolo Santi, Member, IEEE “A Framework for Routing Performance Analysis in Delay Tolerant Networks with Application to Noncooperative Networks" TRANSACTIONS ON PARALLEL AND DISTRIBUTED SYSTEMS, VOL. 23, NO. 1, JANUARY 2012

[7] Yong Li, Student Member, IEEE, Guolong Su, Dapeng Oliver Wu, Senior Member, IEEE,Depeng Jin, Member, IEEE, Li Su, and Lieguang Zeng "The Impact of Node Selfishness on Multicasting in Delay Tolerant Networks". IEEE VOL. 60, NO. 5, JUNE 2011. 